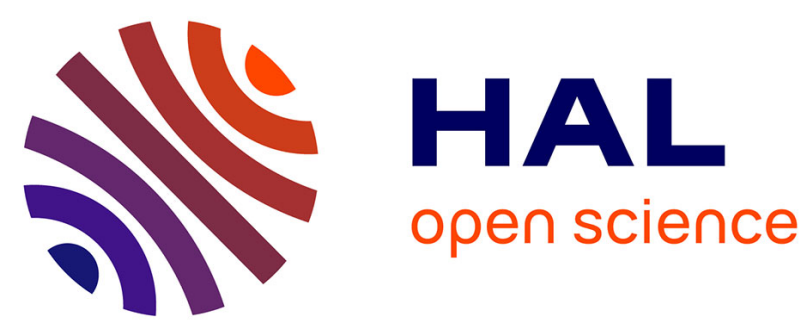

\title{
Acoustic analogue of electromagnetically induced transparency and Autler-Townes splitting in pillared metasurfaces
}

Yabin Jin, Yan Pennec, Bahram Djafari-Rouhani

\section{To cite this version:}

Yabin Jin, Yan Pennec, Bahram Djafari-Rouhani. Acoustic analogue of electromagnetically induced transparency and Autler-Townes splitting in pillared metasurfaces. Journal of Physics D: Applied Physics, 2018, 51 (49), 10.1088/1361-6463/aae4f3 . hal-03183511

\section{HAL Id: hal-03183511 https://hal.science/hal-03183511}

Submitted on 23 Aug 2021

HAL is a multi-disciplinary open access archive for the deposit and dissemination of scientific research documents, whether they are published or not. The documents may come from teaching and research institutions in France or abroad, or from public or private research centers.
L'archive ouverte pluridisciplinaire HAL, est destinée au dépôt et à la diffusion de documents scientifiques de niveau recherche, publiés ou non, émanant des établissements d'enseignement et de recherche français ou étrangers, des laboratoires publics ou privés.

\section{(c)(1)}

Distributed under a Creative Commons Attribution| 4.0 International License 


\title{
Acoustic Analogue of Electromagnetically Induced Transparency and
}

\author{
Autler-Townes Splitting in Pillared Metasurfaces \\ Yabin Jin ${ }^{1, *}$, Yan Pennec ${ }^{2}$, Bahram Djafari-Rouhani ${ }^{2}$ \\ ${ }^{1}$ School of Aerospace Engineering and Applied Mechanics and Institute for Advanced Study, Tongji \\ University, 200092 Shanghai, China \\ ${ }^{2}$ Insitut d'Electronique, de Microélectronique et de Nanotechnologie, UMR CNRS 8520, Université de \\ Lille, 59650 Villeneuve d'Ascq, France \\ *corresponding author: 083623jinyabin@tongji.edu.cn
}

Electromagnetically induced transparency (EIT) and Autler-Townes splitting (ATS) originating from multilevel atomic systems have similar transparency windows in transmission spectra which easily brings confusion in their discrimination, despite the difference in their physical mechanisms. Indeed, Fano interference is involved in EIT but not in ATS. There has been great interest in classic analogues of EIT and ATS in recent years, such as in photonics, plasmonics, optomechanics, however, the acoustic analogue of ATS has been rarely studied. In this work, we propose to investigate these phenomena in a pillared metasurface consisting of two lines of pillars on top of a thin plate. The existence of Fabry-Pérot resonance and the intrinsic resonances of the two lines of pillars act as a three-level atomic system that gives rise to acoustic analogue of EIT and ATS. Since the frequency of Fabry-Pérot resonance can be tuned by controlling the distance between the two lines, the underlying physics, whether Fano interference is involved or not, are quite clear to discriminate them. The realizations of EIT and ATS put forward to control elastic waves for potential applications such as sensing, imaging, filtering.

\section{Introduction}

Phononic crystals[1-6] and acoustic metamaterials[7-10] are artificial acoustic composite materials controlling elastic/acoustic wave in novel ways and receiving large amounts of attention in broad communities. Phononic crystals possess Bragg band gaps resulting from the destructive interference among the inclusions/scatters when working wavelength is in the order of the lattice parameter, with applications like wave guiding[11-13], filtering[14-16], acoustic lensing[17-19] and so on; acoustic metamaterials exhibit hybridization band gaps resulting from local resonances at a larger wavelength (lower frequency than Bragg band gap) and generate negative effective density or/and bulk modulus for negative refraction and super-resolution imaging[20, 21], cloaking[22-24] among others. In the past decade, pillared structures $[25,26]$ that consist of periodic array of pillars on top of a plate have been receiving increasing interest. Indeed, since they can exhibit both Bragg and hybridization band gaps and serve as pillared phononic crystals and pillared acoustic metamaterials, respectively. Therefore they exhibit potentialities for manipulating 
elastic waves in various applications such as superlensing[27], waveguiding[28-30], fluid sensing[31], thermal conductivity control[32-34], topologically protected edge states[35, 36], among others. The intrinsic resonances of pillar-type-scatters in these structures can couple to other modes in the substrate that give rise to Fano resonances[37, 38].

Among various pillared phononic crystals and metamaterials, a new type of structure that consists of a single or a line of pillars on top of a thin plate, also named as pillared metasurface[39,40], is recently proposed, where the intrinsic properties of local resonances of a pillar are deeply investigated. In fact, a pillar can exhibit a monopolar compressional mode and a dipolar bending mode whose frequencies can be tuned by the geometric parameters of the pillar. The resonant modes can be coupled to the Lamb waves in the plate that results in a transmission dip due to the destructive interference between incident wave and scattered wave by the resonant pillar. Fano resonance is found when two dissimilar pillars are introduced in each unit cell. The peak in the asymmetric transmission is always maintained either the coupling of the two pillars is strong or weak[41].

Fano resonances were used to describe asymmetries in the autoionization spectra in atoms, named after the physicist Ugo Fano who firstly explained it in theory as the interference between individual resonances in the continuum[42]. They are also related to electromagnetically induced transparency (EIT)[43, 44] that needs a discrete transition coupled to a continuum, giving rise to a transparency window in the absorption/transmission spectrum. Similar transparency windows can also be found in Autler-Townes splitting (ATS) effect[45] but with essentially a different mechanism[46, 47]: EIT is a result of Fano interference among several transition pathways, while ATS requires field-induced splitting of energy that does not require any Fano interference effect. EIT and ATS have been experimentally observed in quantum[48]/atom systems $[49,50]$ as well as some classic systems such as in photonic crystals[51-53], metamaterials[54], plasmonics[55], optomechanics[56, 57], micro-resonators[46, 5861], which are widely applied for controlling light at room temperature. Since the transparency profiles in absorption/transmission spectrum of EIT and ATS closely resemble each other, they are easy to be confused. Several methods[62-65] are proposed to discriminate between them based on observed absorption or transmission spectra, but not on the physical mechanism behind (such as whether Fano resonance is involved or not). In another classic aspect, acoustic system, Fano resonance and EIT are also investigated in several different structures[66-70]; however, the acoustic analogue of ATS has been rarely reported.

In this work, we propose pillared metasurfaces, two lines of pillars on top of a thin plate, and find Fano interference originated from Fabry-Pérot resonance between the two lines of pillars. By tuning the distance $L$ between the two lines, the frequency of Fabry-Pérot resonance can be easily controlled as the wavelength at Fabry-Pérot resonance is two times the distance $L$. When the frequency of Fabry-Pérot resonance and the resonant frequency of two identical pillars are the same, Fabry-Pérot resonance becomes invisible as a bound state in the continuum. Detuning the resonant frequency of the two pillars, Fabry-Pérot resonance becomes stronger and EIT effect is observed 
due to the destructive interference; on the other hand, when Fabry-Pérot resonance is far beyond the considered resonance frequency domain, ATS effect is observed when the two pillars are strongly coupled. We realize EIT and ATS in these pillared metasurfaces by directly discriminating the essential physical mechanisms to avoid any confusion, rather than employing some fitting-parameter methods from obtained transmission spectra. Like the wide applications in optics or other systems, the realization of EIT and ATS in this work puts forward the control of elastic waves for potential applications such as sensing, imaging, filtering, among others, in micro or nano scale.

\section{Pillared metasurfaces}

We present in Fig. 1 the unit cell of the acoustic metasurface made of two lines of pillars deposited on a thin plate (thickness $e=145 \mu \mathrm{m}$ ) in micro-scale and choose cubic silicon as the material for the whole structure, with elastic constants $c_{11}=166 \mathrm{GPa}$, $c_{12}=64 \mathrm{GPa}, c_{44}=79.6 \mathrm{GPa}$ and density $\rho=2330 \mathrm{~kg} / \mathrm{m}^{3}$. The crystallographic axes [100] and [010] of silicon are chosen parallel to the $x$ and $y$ axes, respectively. We employ finite element method to carry out full wave simulations in frequency domain with time-harmonic condition. The infinite arrangement of two lines of pillars in $y$ axis is considered by applying periodic conditions to the two sides of the unit cell, where $a=200 \mu m$ is the periodicity along $y$ axis. Perfectly marched layers (PML) are added at the two edges in $x$ axis to avoid any wave reflection from the external edges. The fundamental anti-symmetric $\left(\mathrm{A}_{0}\right)$ Lamb wave is excited that is dominated by out of plane component of the displacement $u_{z}$. A far field point T $(1000 \mu m$ away from pillar 2 ) on the surface of the plate after the two lines of pillars is selected to detect $u_{z}$, which is further used to calculate the transmission curves by normalizing to the $u_{z}$ on the same point without the pillars.

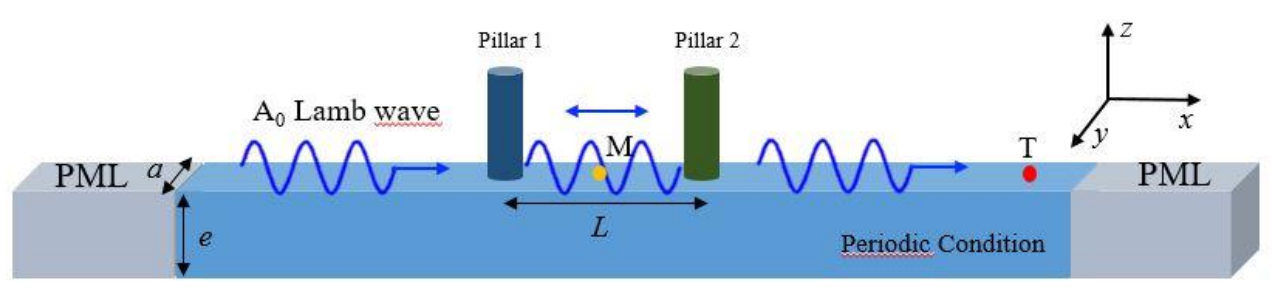

Figure 1. 3D schematic view of the unit cell of the geometric model: two lines of pillars are deposited on top of a thin plate with a thickness $e$. Periodic conditions are applied to the two sides along $y$ axis and perfectly marched layers (grey) are applied to the two edges along $x$ axis. The period is $a$ along $y$ axis and the distance between the two lines of pillars along $x$ axis is $L$. The fundamental anti-symmetric $\left(\mathrm{A}_{0}\right)$ 
Lamb mode wave is excited to propagate towards $x$ direction and is scattered by the two line of pillars before exit. $\mathrm{M}$ is the middle point between the two lines on the surface.

\section{Acoustic analogue of EIT}

EIT refers to the quantum interference of excitation with a three-level atomic system where a narrow transparency windows appears in an opaque region. In this pillared metasurface, the resonance of two pillars together with the Fabry-Pérot resonance act like "three-level atomic system" in acoustics. By detuning the height of the two pillars in order to separate the corresponding dips in the transmission and by adjusting the length $L$ such that the Fabry-Pérot resonance falls exactly between the two dips, one obtains the spectra shown in Fig. 2a for different values of the detuning. In this figure, the distance $L$ is fixed to $230 \mu \mathrm{m}$, the radius of the two pillars is fixed to $25 \mu \mathrm{m}$ and the heights of the pillars are symmetrically detuned around $245 \mu \mathrm{m}$. When $\mathrm{h} 1=\mathrm{h} 2=245 \mu \mathrm{m}$, the Fabry-Pérot resonance coincides with the compressional resonance frequencies of both pillars which means the round-trip phase shift of the wave between the two pillars is added up to $2 \pi$; then a Fabry-Pérot bound state in the continuum (BIC) is formed[71].

When the pillars are in resonant states, the displacement field in the plate can be regarded as the sum of the incident and scattered waves. As a consequence, the scattered wave is obtained as the subtraction between the full transmitted wave and the incident wave. In Fig.2b-d, we show the Nyquist plots of the scattered waves normalized to the incident wave at the far field for three examples of Fig.2a, namely curves in green (h1 $=240 \mu m, \mathrm{~h} 2=250 \mu \mathrm{m})$, cyan $(\mathrm{h} 1=242 \mu \mathrm{m}, \mathrm{h} 2=248 \mu \mathrm{m})$ and blue $(\mathrm{h} 1=\mathrm{h} 2=245 \mu \mathrm{m})$. In this Nyquist plot, if a point locates at the $+x,+y,-x$ or $-y$ axes, it means that the phase of scattered wave with respect to the incident wave at the corresponding frequency is $0, \pi / 2, \pi /-\pi,-\pi / 2$, respectively. When the heights of the two pillars are different from $245 \mu m$ while keeping $L=230 \mu m$, (in another word, the compressional resonance frequencies of the two pillars are different from that of the Fabry-Pérot resonance), the Fabry-Pérot resonance becomes visible in the transmission curve. The inner green and cyan ellipses in Fig.2b\&c show that Fabry-Pérot resonance becomes stronger with increasing height difference as the green inner ellipse is bigger, hence the peak $p$ corresponds to a higher transmission (or weaker scattered field). The inner ellipse cuts the $-x$ axis at $\mathrm{p}$ and $\mathrm{d} 2$, and is out of phase with respect to the incident wave, which gives rise to the peak $\mathrm{p}$ and dip $\mathrm{d} 2$ in transmission in Fig.2a. The frequency of peak $\mathrm{p}$ corresponds to the Fabry-Pérot resonance and keeps unchanged as the spacing distance $\mathrm{L}$ is fixed at $230 \mu \mathrm{m}$. For green case, the position $p$ of the inner ellipse is more close to the origin, so that the transmission peak is more towards 1, being as acoustic analogue of EIT. The transmission dips $\mathrm{d} 1$ and $\mathrm{d} 2$ follow the individual intrinsic resonant frequencies (circle dots in Fig.2a), which is also supported from the vibrating states in the inserts. As a result, the acoustic analogue of EIT involves Fabry-Pérot resonance that contributes to the peak and pillar's intrinsic resonances that contribute to the two dips. 
(a)

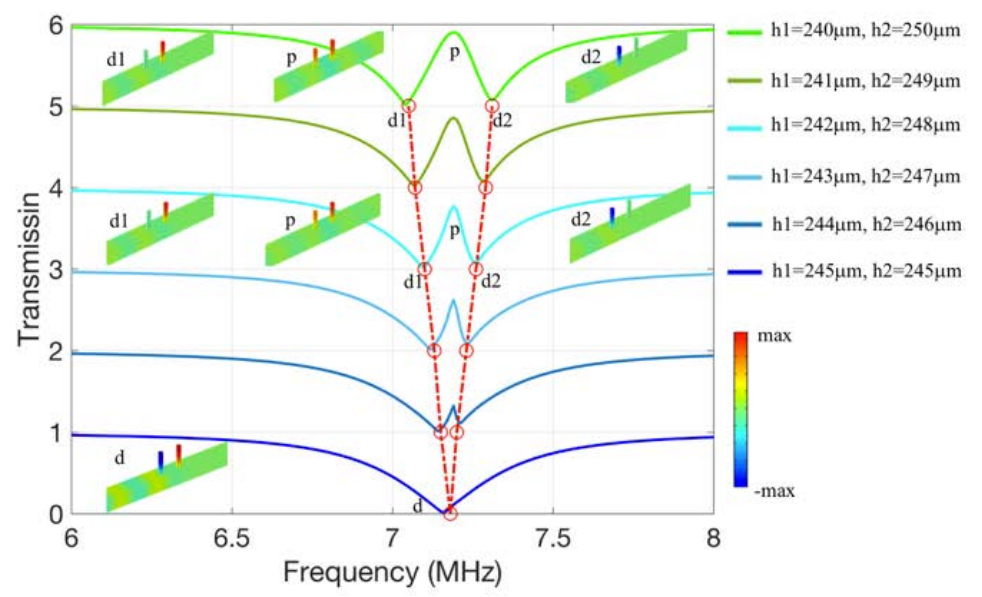

(b)

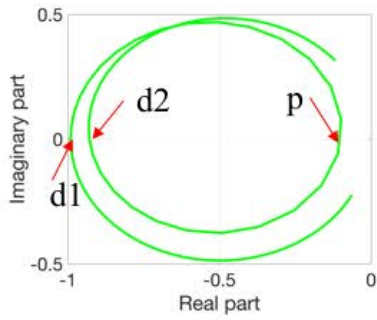

(c)

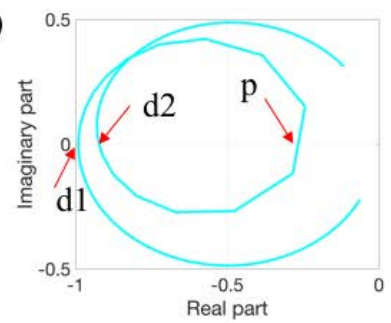

(d)

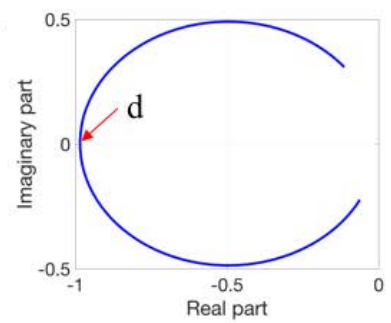

Figure 2. Acoustic analogue of EIT: (a) when L=230 $\mu \mathrm{m}$, Fabry-Pérot resonance becomes stronger if the dissimilarity of height in two lines of pillars increases, which results in a sharp transparent window in transmission. Real part of the displacement field $u_{z}$ at dips and peak are shown as insert. In all inserts, pillars 1 and 2 are at left and right, respectively. The circle dots represent the individual intrinsic resonant frequencies of pillars; Lower panel: Nyquist plot of scattered wave at far field for the green (h1 $=240 \mu m$, $\mathrm{h} 2=250 \mu \mathrm{m})$, cyan $(\mathrm{h} 1=242 \mu \mathrm{m}, \mathrm{h} 2=248 \mu \mathrm{m})$ and blue $(\mathrm{h} 1=\mathrm{h} 2=245 \mu \mathrm{m})$ curves shown in (b), (c) and (d), respectively. The radius of the two pillars is equal to $25 \mu \mathrm{m}$.

\section{Fano resonance}

In this section, we will discuss how EIT deviates to ATS in this pillared metasurface. Before considering the scattering by the two lines of pillars, we first study the case of a single line of pillars to give a basic view of transmission properties. In this calculation, the radius of the pillars is $r=25 \mu \mathrm{m}$ and their height $h=245 \mu \mathrm{m}$. As shown by the red line in Fig.3, a transmission dip appears in the frequency domain [6, 8] MHz associated with the monopolar (compressional) resonance of this pillar that locates at $f=7.19 \mathrm{MHz}$ (see the real part of $u_{z}$ ), well isolated from other intrinsic resonances in this frequency domain. The compressional mode of the pillar can be excited by the incident $\mathrm{A}_{0}$ Lamb wave dominated by the displacement component $u_{z}$ and emits the same $\mathrm{A}_{0}$ Lamb wave. When the incident and emitted $A_{0}$ Lamb waves are out of phase, destructive interference happens and results in such transmission dip in the spectrum.

Considering two lines of such identical pillars separated by a distance $L$, the transmission properties versus distance $L$ are plotted in Fig.3 with $L$ varying from $100 \mu m$ to $350 \mu m$. One can observe that the transmission spectra are more complex than 
that of a single line case.

Fabry-Pérot interference occurs when the wavelength is 2 times the distance $L$. At $L=100 \mu m$, Fabry-Pérot resonance is much above the frequency domain [6-8] MHz, so that it is the coupling of the two identical pillars that gives a splitting in the transmission with two dips. When $L$ increases to $200 \mu \mathrm{m}$, Fabry-Pérot resonance red-shifts into the frequency domain [6-8] MHz and interacts with one of the resonance frequencies, hence producing an asymmetric Fano type resonance. Then at $L=230 \mu m$, the Fabry-Pérot resonance coincides exactly with the zero of transmission of both pillars, and the transmission spectrum containing a single dip reveals the case of a bound state in the continuum (BIC)[71]. When $L$ continues to increase, the Fabry-Pérot resonance moves to much lower frequency range and the transmission spectrum displays a broad dip characteristic of a low coupling between the two pillars. The inserts in Fig.3 also indicate that the two pillars couple each other when $L<230 \mu \mathrm{m}$, behave as out of phase resonant vibration when $L=230 \mu m$, and has very weak coupling when $L$ becomes much higher than $230 \mu m$.

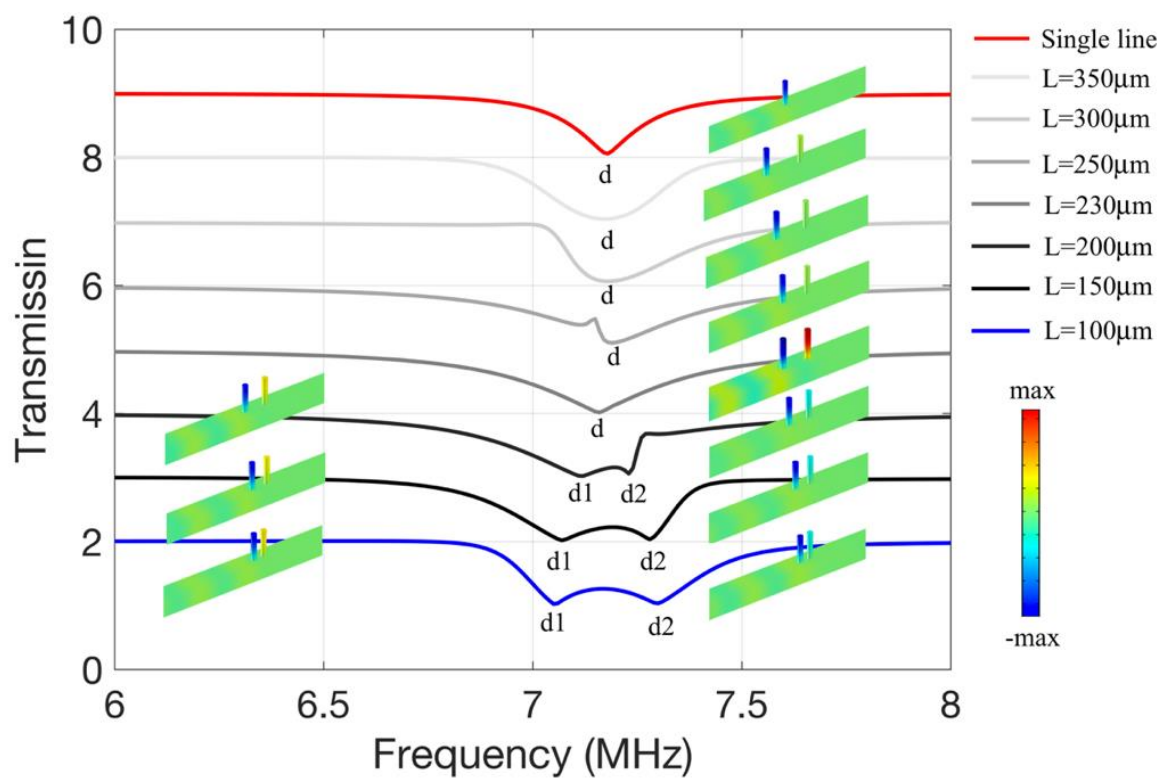

Figure 3. Transmission versus frequency for a single line of pillars (red line) or two lines of the identical pillars with different distance $L$. The radius $r$ of the pillar is $25 \mu \mathrm{m}$ and the height $h$ of the pillar is $245 \mu \mathrm{m}$. Real part of the displacement fields $u_{z}$ at the dip d1 and $\mathrm{d} 2$ are respectively shown at the left and at the right. In inserts with two pillars, pillars 1 and 2 are at left and right, respectively.

In order to better understand how the Fabry-Pérot resonance changes around $L=230 \mu \mathrm{m}$, we take a smaller step in $L$, as $225 \mu \mathrm{m}, 228 \mu \mathrm{m}, 229 \mu \mathrm{m}, 230 \mu \mathrm{m}, 232 \mu \mathrm{m}$, and $235 \mu \mathrm{m}$, and show the transmission spectra in a zoom-in frequency domain $[7,7.5] \mathrm{MHz}$ in Fig. $4 a$. When $L=225 \mu \mathrm{m}, 232 \mu \mathrm{m}$ and $235 \mu \mathrm{m}$, there is a slight peak and dip at the right side of the main dip; however it's hardly to observe them for $L=228 \mu \mathrm{m}, 229 \mu \mathrm{m}, 230 \mu \mathrm{m}$. as the Fabry-Pérot resonance becomes a BIC. We further select a middle point M on the surface of the plate between the two lines and detect the amplitude of $u_{z}$ on this M point. Normalized to the $u_{z}$ of same position $\mathrm{M}$ without pillars, the relative amplitude 
is plotted as Fig. $4 b$ that clearly shows the evolution of an asymmetric profile. This profile first decreases to almost zero at $L=229 \mu \mathrm{m}$ then increases again when increase $L$ from $225 \mu \mathrm{m}$ to $235 \mu \mathrm{m}$. In addition, the profile suffers a phase change when $L$ transverses $229 \mu \mathrm{m}$ manifested by the fact the dip follows the peak or vice-versa.
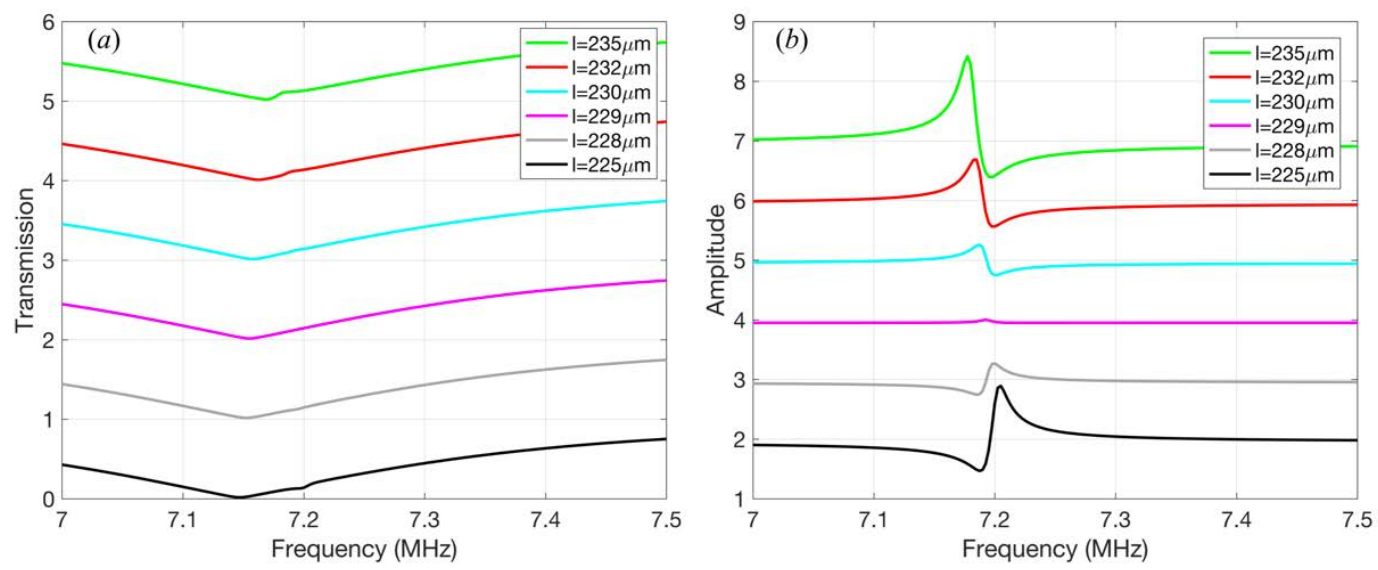

Figure 4. (a)Transmission versus frequency when $L=225 \mu \mathrm{m}, 228 \mu \mathrm{m}, 229 \mu \mathrm{m}, 230 \mu \mathrm{m}, 232 \mu \mathrm{m}$, and $235 \mu \mathrm{m}$; (b) Amplitude of the displacement field $u_{z}$ at the point $\mathrm{M}$ at the middle of the two pillars with the same values of $L$ as in $(a)$. The asymmetric profile disappears when the Fabry-Pérot resonance is in coincide with pillar's compressional mode at $L=229 \mu \mathrm{m}$.

In Fig.5a, Nyquist plots of the scattered wave normalized to the incident wave at the M point are shown for $L=200 \mu \mathrm{m}, 229 \mu \mathrm{m}$, and $250 \mu \mathrm{m}$. They exhibit a blue ellipse in the positive half $y$ space, a dot at origin, and a green ellipse in the negative half $y$ space, respectively. The blue and green ellipses stand for scattered waves by FabryPérot resonance and the pink dot at origin shows that Fabry-Pérot resonance is invisible at $L=229 \mu \mathrm{m}$. Then in Fig.5b, we show the Nyquist plot of the scattered waves normalized to the incident wave at the far field point that is detected for transmission calculation. Following the explanation of Nyquist plot of the normalized scattered wave in Section 3, for $L=229 \mu \mathrm{m}$, the scattered field at b point is out of phase with respect to the incident field and its amplitude is almost the same, a slightly smaller; consequently, the transmitted field almost vanishes while it remains in phase with the incident field.

For $L=200 \mu m$ and $250 \mu m$, the Nyquist plots of scattered waves at far field in Fig.5b show protruding shape with respect to the pink ellipse, which associates with visible Fabry-Pérot resonance. The blue and green protruding curves cut $-x$-axis at $a$ point (very close to $x=-1$ ) and c point (about $\mathrm{x}=-1.4$ ), respectively. The $a$ point corresponds to the 0 phase in Fig. $4 \mathrm{c}$ as the case of point $\mathrm{b}$. The $\mathrm{x}=-1.4$ at $\mathrm{c}$ point means the amplitude of scattered wave is 1.4 times that of incident wave while they are out of phase, so that a new transmission peak appears (as seen in Fig.3) and the phase is $\pi$ or $-\pi$. The real part of $u_{z}$ at a, b, and c points are shown in Fig. $5 \mathrm{~d}$. The real part field at $L=229 \mu m$ behaves as a transition of those at $L=200 \mu m$ and $250 \mu m$ when destructive interference happens. 

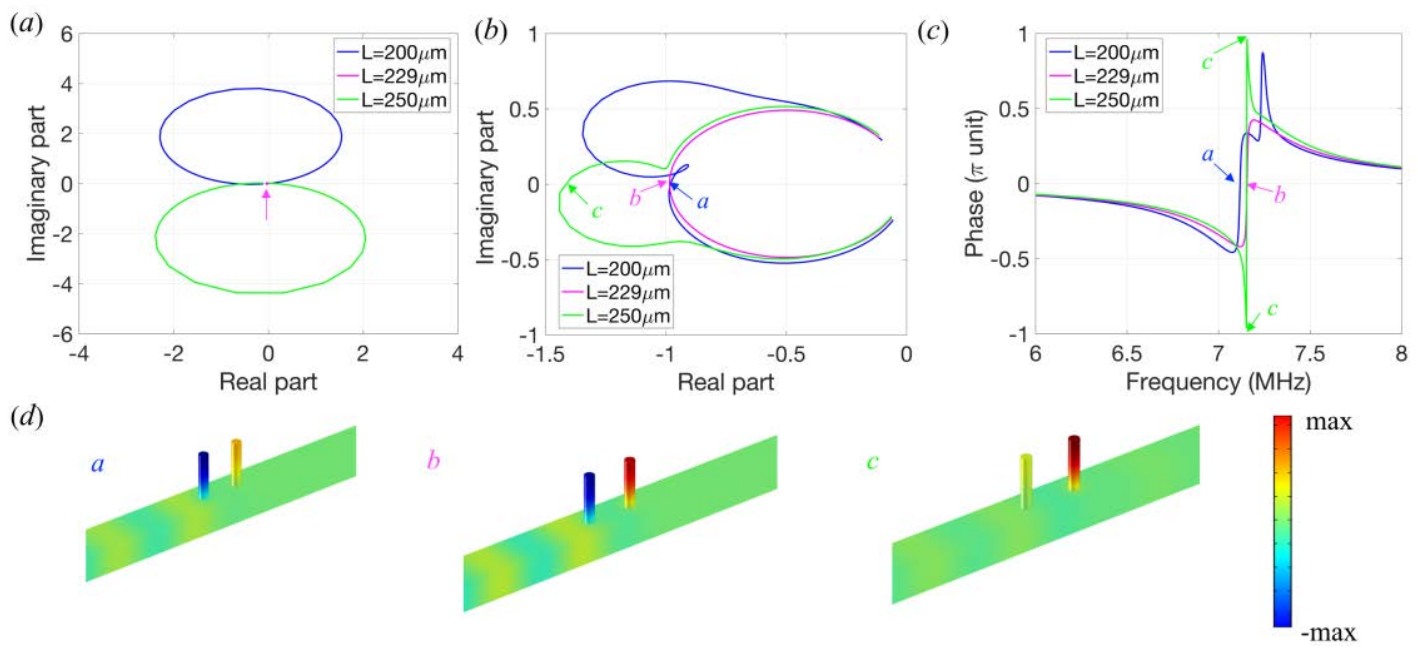

Figure 5.(a) Nyquist plot of the scattered waves at $\mathrm{M}$ point in the frequency range $[6,8] \mathrm{MHz}$ for $\mathrm{L}=200 \mu \mathrm{m}, 229 \mu \mathrm{m}$, and $250 \mu \mathrm{m}$. The arrow shows that for $\mathrm{L}=229 \mu \mathrm{m}$, the Nyquist plot is a point at origin that means there is no scattered wave induced by Fabry-Pérot resonance;(b) similar Nyquist plot of scattered waves but for a point at far field after the two lines of pillars. Point $\mathrm{a}, \mathrm{b}$, and c cuts the $-x$ axis for $\mathrm{L}=200 \mu \mathrm{m}, 229 \mu \mathrm{m}$, and $250 \mu \mathrm{m}$, respectively; (c) phase of transmitted wave at this far field point. Point a, b, and c are at the same frequencies as in (b); (d) real part of the displacement field $u_{z}$ for point $\mathrm{a}, \mathrm{b}$ and c. Pillars 1 and 2 are at left and right, respectively.

\section{What is and what is not acoustic analogue of ATS?}

ATS requires no Fano interference, which is still induced from the coupling of the resonators. Therefore, for two separated transmission dips, if there is no coupling between the two resonators (which means the dips are very close to those of the isolated resonators), it's not an ATS.

In section 4 , we showed that for $L=[100,350] \mu m$, coupling effect between the two pillars happens when $L$ is smaller than $230 \mu m$ while very weak coupling happens when $L$ is bigger than $230 \mu \mathrm{m}$. In Fig.6, the Nyquist plot of scattered wave at far field point within $[6,8] \mathrm{MHz}$ for $L=100 \mu \mathrm{m}$ and $350 \mu \mathrm{m}$ are plotted as black and blue curve, respectively, where the two pillars are identical (no detuning frequency). Fabry-Pérot resonance is beyond the frequency domain $[6,8] \mathrm{MHz}$ for both cases. For $L=100 \mu \mathrm{m}$, two ellipses cross with an inner close-shape curve that cuts $-x$-axis three times close to $x=-1$, giving rise to two transmission dips as seen in Fig.3, as an ATS. For $L=350 \mu m$, the two ellipses merge as a heart shape without any interaction, and cuts $-x$-axis only one time at a point a bit exceeding $x=-1$, so that there is only one transmission dip whose phase is $\pi$ or $-\pi$. 


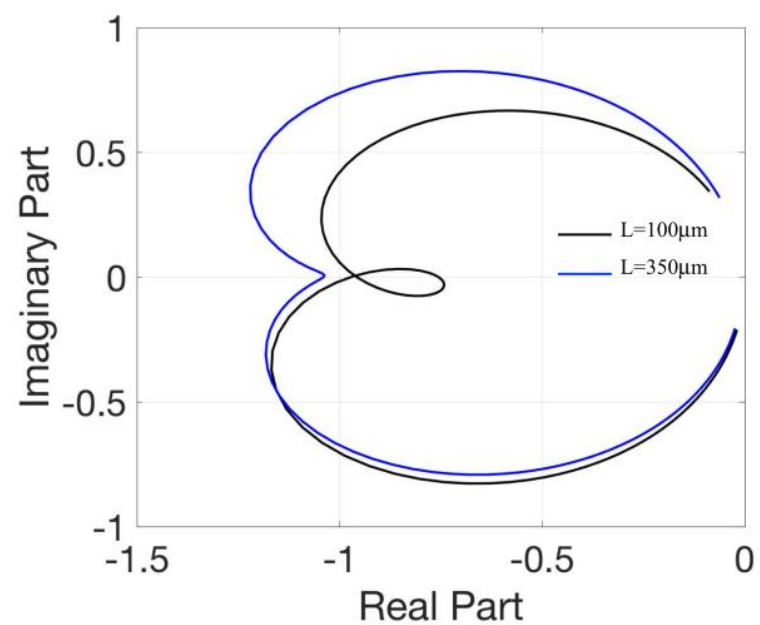

Figure.6 Nyquist plot of scattered wave at far field within $[6,8] \mathrm{MHz}$ for $\mathrm{L}=100 \mu \mathrm{m}$ (black) and $350 \mu \mathrm{m}$ (blue).

For $L=100 \mu m$, we keep the height of the first pillar fixed as $\mathrm{h} 1=245 \mu \mathrm{m}$ and make a sweep in the height of the second pillar from $220 \mu \mathrm{m}$ to $270 \mu \mathrm{m}$. The frequencies of the two resonators are then detuned with respect to each other. The evolution of individual resonant frequency when the height changes from $220 \mu \mathrm{m}$ to $270 \mu \mathrm{m}$ is also plotted as red circle-dotted line, whereas the resonant frequency for individual pillar with height as $245 \mu \mathrm{m}$ is plotted as blue circle-dotted line. From the transmission spectra in Fig.7, the ATS exhibits avoided crossing effect. When $\mathrm{h} 1=\mathrm{h} 2=245 \mu m$, the two dips in the transmission deviate the most from the individual values, showing the strongest coupling between the two pillars. When h2 is larger or smaller than $245 \mu \mathrm{m}$, such deviation decreases and the two dips get more close to the individual values, showing a weakening of the coupling effect. Therefore, when $\mathrm{h} 1=\mathrm{h} 2=245 \mu \mathrm{m}$ or $\mathrm{h} 2$ is close to $245 \mu \mathrm{m}$, such as $\mathrm{h} 2=240 \mu \mathrm{m} / 250 \mu \mathrm{m}$, it's ATS because there is no Fano interference effect involved and the coupling effect between the two pillars are strong. When h2 is far away from $245 \mu \mathrm{m}$, the transmission dips result from individual resonances without coupling effect, so that it's not ATS. 


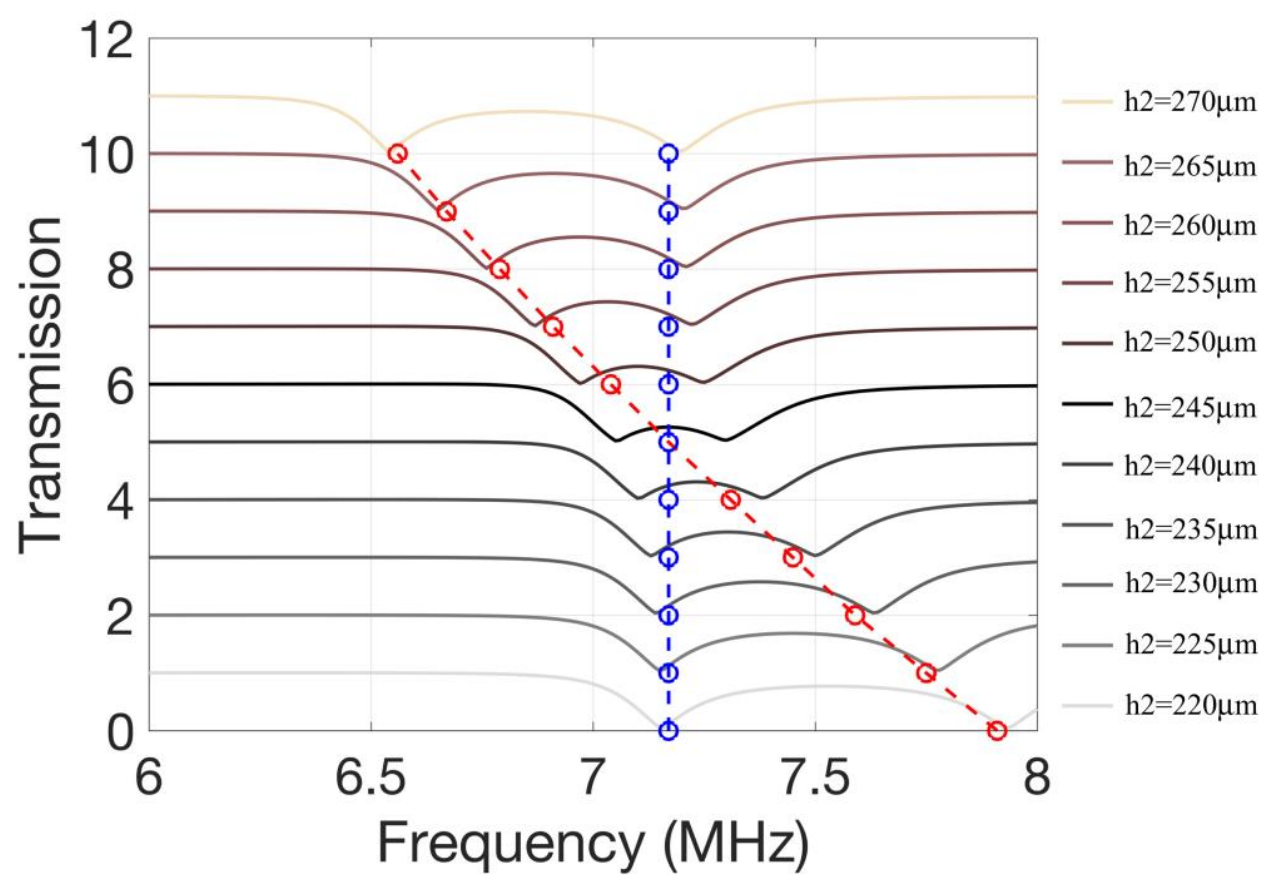

Figure.7 Avoided crossing ATS. For L $=100 \mu m$, the height of the first pillar h1 is fixed to $245 \mu m$, and the height of the second pillar h2 is gradually changed from $220 \mu \mathrm{m}$ to $270 \mu \mathrm{m}$. The effect of the frequency detuning of the coupled resonant modes on ATS exhibits avoided crossing. The evolution of individual resonant frequency when the height changes from $220 \mu \mathrm{m}$ to $270 \mu \mathrm{m}$ is also plotted as red circle-dotted line whereas the resonant frequency for individual pillar with height as $245 \mu \mathrm{m}$ is plotted as blue circledotted line.

For $\mathrm{L}=350 \mu \mathrm{m}$, we make a similar sweep in the height of the second pillar from $220 \mu \mathrm{m}$ to $270 \mu \mathrm{m}$, while keeping h1 fixed. The red circle-dotted line and blue circledotted line show the same individual resonant frequency as in Fig.7. One can clearly see from the upper panel of Fig.8, the two transmission dips exactly follow the individual red and blue circle values for each h2 case. The moving transmission dip goes crossing the fixed dip, as the red circle-dotted line crosses the blue circle-dotted line. It means that there is no coupling between the two pillars for all cases in the upper panel of Fig.8, so that although there are two dips in transmission and no Fano interference, they are not ATS. We also plot such kind of figure for an intermediary value of $\mathrm{L}$ such as $\mathrm{L}=180 \mu m$ (lower panel of Fig.8). Comparing with $\mathrm{L}=350 \mu \mathrm{m}$, the two pillars have coupling effect to split the transmission into two dips when $\mathrm{h} 2=\mathrm{h} 1=245 \mu \mathrm{m}$. However, coupling effect quickly weakens when $\mathrm{h} 2$ becomes differ from $\mathrm{h} 1$. Even for $\mathrm{h} 2=240 \mu \mathrm{m}$ or $250 \mu \mathrm{m}$, the two dips almost follows their individual resonant frequencies. 

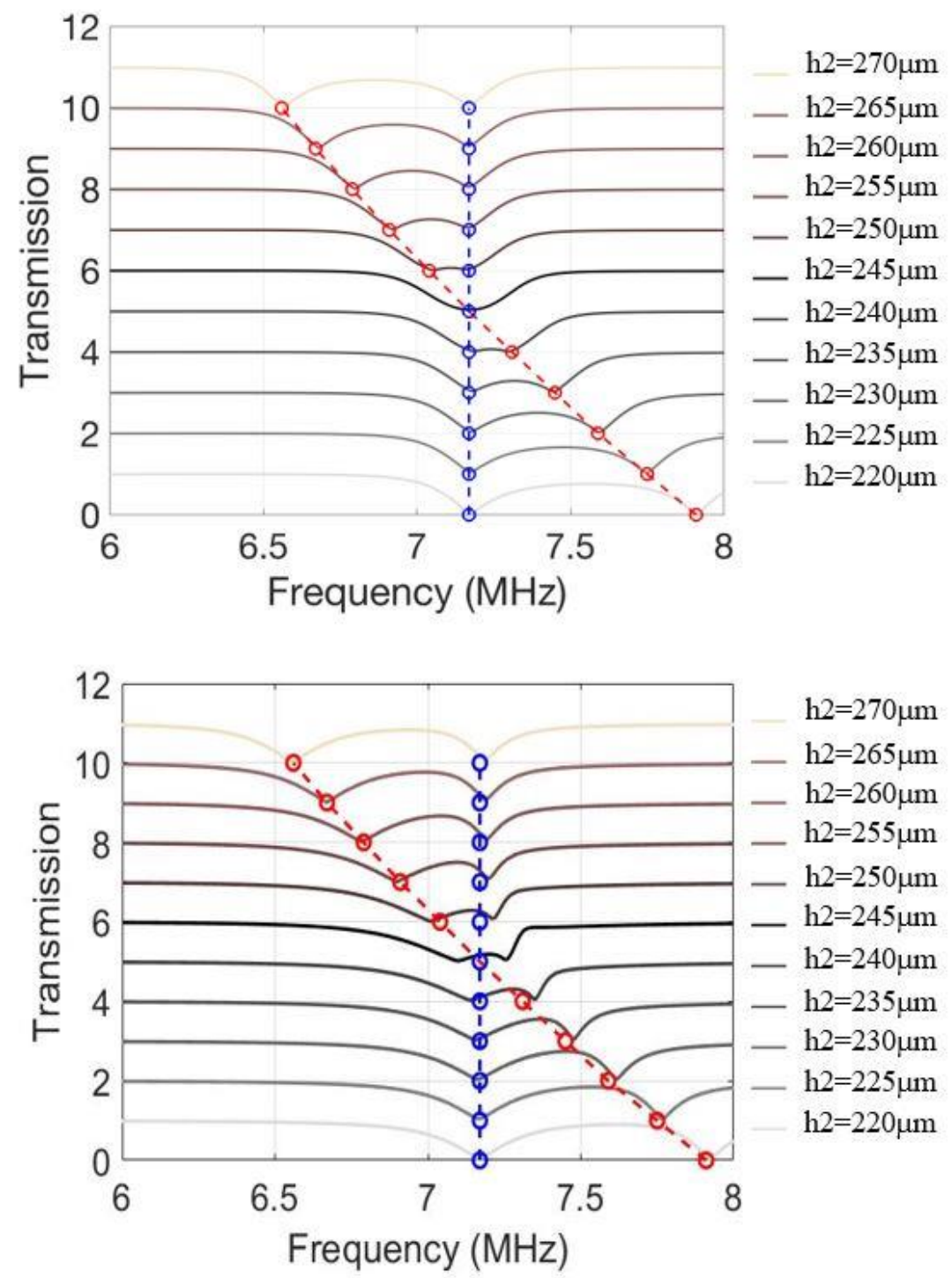

Figure 8. Same as in Fig. 7 when $\mathrm{L}=350 \mu m$ (upper panel) and $\mathrm{L}=180 \mu m$ (lower panel). Increasing the difference in height of the two lines of pillars (detuning the frequencies of the two resonators), the transmission dips follow their corresponding individual resonant frequency in the upper panel. For moderate case in the lower panel, the coupling effect of the two pillars only remains when h2 equals or very close to $245 \mu \mathrm{m}$. Even when $\mathrm{h} 2=240 \mu \mathrm{m}$ or $250 \mu \mathrm{m}$, the coupling effect is too weak that the transmission dips almost follow their individual resonant frequencies.

\section{Summary}

In this work, we realized acoustic analogue of EIT and ATS in pillared metasurfaces, especially since the acoustic analogue of ATS was rarely reported in literature[72]. We constructed a metasurface consisting of two lines of pillars separated by a distance $L$ where a Fabry-Pérot resonance can appear between the two lines at a wavelength which is two times the distance $L$. At a specific case $L=230 \mu m$, Fabry-Pérot resonance and pillar's compressional mode have the same frequency, Fabry-Pérot 
resonance has zero width and becomes invisible, as a BIC. At this $L$, acoustic analogue of EIT was realized by making the heights of the two pillars slightly different. In that case, the Fabry-Pérot resonance becomes visible and stronger and appears as a transparency windows between two dips in the transmission. In contrast, the FabryPérot resonance shifts beyond the working band when $L=100 \mu \mathrm{m}$ or $L=350 \mu \mathrm{m}$, so that no Fano interference happens. ATS is induced by the strong coupling between two resonators. It is found that for $L=100 \mu m$ only when the heights of the two pillars are the same or very close, the coupling effect is strong and the two transmission dips are ATS; when the two heights are far away from each other, the transmission dips are very close to those resulting from individual resonances, so they are not ATS. For $L=350 \mu \mathrm{m}$, similar reason as no coupling effect involves, the two transmission dips are not ATS.

We realized and distinguished acoustic analogue of EIT and ATS by the essential mechanism, whether Fano interference is involved. Moreover, we made it clear what is ATS or not by discriminating whether strong coupling effect happens. The realization of EIT and ATS in acoustics can be applied to control elastic waves for potential applications such as sensing, imaging, filtering.

Acknowledgments

Y.J. acknowledges a start-up fund from Tongji University.

\section{Reference}

1. Kushwaha, M.S., et al., Acoustic band structure of periodic elastic composites. Physical review letters, 1993. 71(13): p. 2022.

2. Sigalas, M. and E.N. Economou, Band structure of elastic waves in two dimensional systems. Solid state communications, 1993. 86(3): p. 141-143.

3. Pennec, Y., et al., Two-dimensional phononic crystals: Examples and applications. Surface Science Reports, 2010. 65(8): p. 229-291.

4. Hussein, M.I., M.J. Leamy, and M. Ruzzene, Dynamics of phononic materials and structures: Historical origins, recent progress, and future outlook. Applied Mechanics Reviews, 2014. 66(4): p. 040802.

5. Ge, H., et al., Breaking the Barriers: Advances in Acoustic Functional Materials. National Science Review, 2017.

6. Dobrzynski, L., et al., Phononics: Interface Transmission Tutorial Book Series. 2017: Elsevier.

7. Liu, Z., et al., Locally resonant sonic materials. Science, 2000. 289(5485): p. 1734-1736.

8. Ma, G. and P. Sheng, Acoustic metamaterials: From local resonances to broad horizons. Science advances, 2016. 2(2): p. e1501595.

9. Cummer, S.A., J. Christensen, and A. Alù, Controlling sound with acoustic metamaterials. Nature Reviews Materials, 2016. 1: p. 16001.

10. Craster, R.V. and S. Guenneau, Acoustic metamaterials: Negative refraction, imaging, lensing and cloaking. Vol. 166. 2012: Springer Science \& Business Media.

11. Kafesaki, M., M. Sigalas, and N. Garcia, Frequency modulation in the transmittivity of wave guides in elastic-wave band-gap materials. Physical Review Letters, 2000. 85(19): p. 4044.

12. Khelif, A., et al., Guiding and bending of acoustic waves in highly confined phononic crystal 
waveguides. Applied physics letters, 2004. 84(22): p. 4400-4402.

13. Khelif, A., et al., Two-dimensional phononic crystal with tunable narrow pass band: Application to a waveguide with selective frequency. Journal of applied physics, 2003. 94(3): p. 1308-1311.

14. Pennec, Y., et al., Tunable filtering and demultiplexing in phononic crystals with hollow cylinders. Physical Review E, 2004. 69(4): p. 046608.

15. Wu, T.-T., L.-C. Wu, and Z.-G. Huang, Frequency band-gap measurement of two-dimensional air/silicon phononic crystals using layered slanted finger interdigital transducers. Journal of Applied Physics, 2005. 97(9): p. 094916.

16. Qiu, C., et al., Mode-selecting acoustic filter by using resonant tunneling of two-dimensional double phononic crystals. Applied Physics Letters, 2005. 87(10): p. 104101.

17. Lin, S.-C.S., et al., Gradient-index phononic crystals. Physical Review B, 2009. 79(9): p. 094302.

18. Jin, Y., et al., Simultaneous control of the $S O$ and $A 0$ Lamb modes by graded phononic crystal plates. Journal of Applied Physics, 2015. 117(24): p. 244904.

19. Jin, Y., et al., Gradient index devices for the full control of elastic waves in plates. Scientific reports, 2016. 6: p. 24437.

20. Zhang, S., L. Yin, and N. Fang, Focusing ultrasound with an acoustic metamaterial network. Physical review letters, 2009. 102(19): p. 194301.

21. Kaina, N., et al., Negative refractive index and acoustic superlens from multiple scattering in single negative metamaterials. Nature, 2015. 525(7567): p. 77.

22. Torrent, D. and J. Sánchez-Dehesa, Acoustic cloaking in two dimensions: a feasible approach. New Journal of Physics, 2008. 10(6): p. 063015.

23. Cummer, S.A. and D. Schurig, One path to acoustic cloaking. New Journal of Physics, 2007. 9(3): p. 45.

24. Chen, H. and C. Chan, Acoustic cloaking in three dimensions using acoustic metamaterials. Applied physics letters, 2007. 91(18): p. 183518.

25. Pennec, Y., et al., Low-frequency gaps in a phononic crystal constituted of cylindrical dots deposited on a thin homogeneous plate. Physical Review B, 2008. 78(10): p. 104105.

26. Wu, T.-T., et al., Evidence of complete band gap and resonances in a plate with periodic stubbed surface. Applied Physics Letters, 2008. 93(11): p. 111902.

27. Zhao, J., B. Bonello, and O. Boyko, Focusing of the lowest-order antisymmetric Lamb mode behind a gradient-index acoustic metalens with local resonators. Physical Review B, 2016. 93(17): p. 174306.

28. Jin, Y., et al., Tunable waveguide and cavity in a phononic crystal plate by controlling whispering-gallery modes in hollow pillars. Physical Review B, 2016. 93(5): p. 054109.

29. Jin, Y., et al., Phononic crystal plate with hollow pillars connected by thin bars. Journal of Physics D: Applied Physics, 2016. 50(3): p. 035301.

30. Guo, Y., M. Hettich, and T. Dekorsy, Guiding of elastic waves in a two-dimensional graded phononic crystal plate. New Journal of Physics, 2017. 19(1): p. 013029.

31. Jin, Y., et al., Phononic crystal plate with hollow pillars actively controlled by fluid filling. Crystals, 2016. 6(6): p. 64.

32. Davis, B.L. and M.I. Hussein, Nanophononic metamaterial: Thermal conductivity reduction by local resonance. Physical review letters, 2014. 112(5): p. 055505.

33. Xiong, S., et al., Blocking phonon transport by structural resonances in alloy-based 
nanophononic metamaterials leads to ultralow thermal conductivity. Physical review letters, 2016. 117(2): p. 025503.

34. Anufriev, R. and M. Nomura, Heat conduction engineering in pillar-based phononic crystals. Physical Review B, 2017. 95(15): p. 155432.

35. Vila, J., R.K. Pal, and M. Ruzzene, Observation of topological valley modes in an elastic hexagonal lattice. Physical Review B, 2017. 96(13): p. 134307.

36. Jin, Y., D. Torrent, and B. Djafari-Rouhani, Robustness of Conventional and Topologically Protected Edge States in Phononic Crystal Plates. Physical Review B, 2018, 98: p. 054307.

37. Pennec, Y., et al., Perpendicular transmission of acoustic waves between two substrates connected by sub-wavelength pillars. New Journal of Physics, 2012. 14(7): p. 073039.

38. Nardi, D., et al., Probing thermomechanics at the nanoscale: impulsively excited pseudosurface acoustic waves in hypersonic phononic crystals. Nano letters, 2011. 11(10): p. 4126-4133.

39. Jin, Y., et al., Pillar-type acoustic metasurface. Physical Review B, 2017. 96(10): p. 104311.

40. $\quad$ Oudich, M., et al., Rayleigh Waves in Phononic Crystal Made of Multilayered Pillars: Confined Modes, Fano Resonances, and Acoustically Induced Transparency. Physical Review Applied, 2018. 9(3): p. 034013.

41. Jin, Y., et al., Tunable Fano resonances of Lamb modes in a pillared metasurface. Journal of Physics D: Applied Physics, 2017. 50(42): p. 425304.

42. Fano, U., Effects of configuration interaction on intensities and phase shifts. Physical Review, 1961. 124(6): p. 1866.

43. Fleischhauer, M., A. Imamoglu, and J.P. Marangos, Electromagnetically induced transparency: Optics in coherent media. Reviews of modern physics, 2005. 77(2): p. 633.

44. Boller, K.-J., A. Imamoğlu, and S.E. Harris, Observation of electromagnetically induced transparency. Physical Review Letters, 1991. 66(20): p. 2593.

45. Autler, S.H. and C.H. Townes, Stark effect in rapidly varying fields. Physical Review, 1955. 100(2): p. 703.

46. Peng, B., et al., What is and what is not electromagnetically induced transparency in whispering-gallery microcavities. Nature communications, 2014. 5: p. 5082.

47. Caselli, N., et al., Generalized Fano lineshapes reveal exceptional points in photonic molecules. Nature communications, 2018. 9(1): p. 396.

48. Ahmed, E., et al., Quantum control of the spin-orbit interaction using the Autler-Townes effect. Physical review letters, 2011. 107(16): p. 163601.

49. Mücke, M., et al., Electromagnetically induced transparency with single atoms in a cavity. Nature, 2010. 465(7299): p. 755.

50. Lukin, M., et al., Spectroscopy in dense coherent media: line narrowing and interference effects. Physical review letters, 1997. 79(16): p. 2959.

51. Yang, X., et al., All-optical analog to electromagnetically induced transparency in multiple coupled photonic crystal cavities. Physical review letters, 2009. 102(17): p. 173902.

52. Wei, B. and S. Jian, Objectively discriminating the optical analogy of electromagnetically induced transparency from Autler-Townes splitting in a side coupled graphene-based waveguide system. Journal of Optics, 2017. 19(11): p. 115001.

53. Mouadili, A., et al., Electromagnetically induced absorption in detuned stub waveguides: a simple analytical and experimental model. Journal of Physics: Condensed Matter, 2014. 26(50): p. 505901. 
54. Papasimakis, N., et al., Metamaterial analog of electromagnetically induced transparency. Physical Review Letters, 2008. 101(25): p. 253903.

55. Zhang, S., et al., Plasmon-induced transparency in metamaterials. Physical Review Letters, 2008. 101(4): p. 047401.

56. Weis, S., et al., Optomechanically induced transparency. Science, 2010. 330(6010): p. 15201523.

57. Dong, C., et al., Optomechanical dark mode. Science, 2012. 338(6114): p. 1609-1613.

58. $\mathrm{Xu}, \mathrm{Q}$., et al., Experimental realization of an on-chip all-optical analogue to electromagnetically induced transparency. Physical review letters, 2006. 96(12): p. 123901.

59. Totsuka, K., N. Kobayashi, and M. Tomita, Slow light in coupled-resonator-induced transparency. Physical review letters, 2007. 98(21): p. 213904.

60. Li, B.-B., et al., Experimental controlling of Fano resonance in indirectly coupled whisperinggallery microresonators. Applied Physics Letters, 2012. 100(2): p. 021108.

61. Maleki, L., et al., Tunable delay line with interacting whispering-gallery-mode resonators. Optics letters, 2004. 29(6): p. 626-628.

62. Anisimov, P.M., J.P. Dowling, and B.C. Sanders, Objectively discerning Autler-Townes splitting from electromagnetically induced transparency. Physical review letters, 2011. 107(16): p. 163604.

63. Abi-Salloum, T.Y., Electromagnetically induced transparency and Autler-Townes splitting: Two similar but distinct phenomena in two categories of three-level atomic systems. Physical Review A, 2010. 81(5): p. 053836 .

64. Anisimov, P. and O. Kocharovskaya, Decaying-dressed-state analysis of a coherently driven three-level 1 system. Journal of Modern Optics, 2008. 55(19-20): p. 3159-3171.

65. Giner, L., et al., Experimental investigation of the transition between Autler-Townes splitting and electromagnetically-induced-transparency models. Physical Review A, 2013. 87(1): p. 013823.

66. Hou, Q., et al., Electromagnetically induced acoustic wave transparency in a diamond mechanical resonator. JOSA B, 2016. 33(11): p. 2242-2250.

67. Liu, F., et al., Acoustic analog of electromagnetically induced transparency in periodic arrays of square rods. Physical Review E, 2010. 82(2): p. 026601.

68. Goffaux, C., et al., Evidence of Fano-like interference phenomena in locally resonant materials. Physical review letters, 2002. 88(22): p. 225502.

69. Liu, F., et al., Tunable transmission spectra of acoustic waves through double phononic crystal slabs. Applied Physics Letters, 2008. 92(10): p. 103504.

70. El Boudouti, E., et al., Transmission gaps and Fano resonances in an acoustic waveguide: analytical model. Journal of Physics: Condensed Matter, 2008. 20(25): p. 255212.

71. Hsu, C., et al., Bound states in the continuum. Nature Reviews Materials, 2016. 1(9): p. 16048.

72. Whiteley, S.J., et al., Coherent control of spins with Gaussian acoustics. arXiv preprint arXiv:1804.10996, 2018. 